\title{
Archéopages
}

Archéopages

Archéologie et société

Hors-série 2 | 2010

Archéologie sans frontières

\section{Programme d'étude quadriennal des fortifications médiévales de Césarée}

Jocelyn Martineau, Jean Mesqui et Nicolas Faucherre

\section{(2) OpenEdition}

1 Journals

Édition électronique

URL : https://journals.openedition.org/archeopages/767

DOI : 10.4000/archeopages.767

ISSN : 2269-9872

Éditeur

INRAP - Institut national de recherches archéologiques préventives

Édition imprimée

Date de publication : 1 octobre 2010

Pagination : 50-57

ISSN : $1622-8545$

\section{Référence électronique}

Jocelyn Martineau, Jean Mesqui et Nicolas Faucherre, « Programme d'étude quadriennal des

fortifications médiévales de Césarée ", Archéopages [En ligne], Hors-série 2 | 2010, mis en ligne le 01 octobre 2010, consulté le 23 février 2023. URL : http://journals.openedition.org/archeopages/767 ;

DOI : https://doi.org/10.4000/archeopages.767 

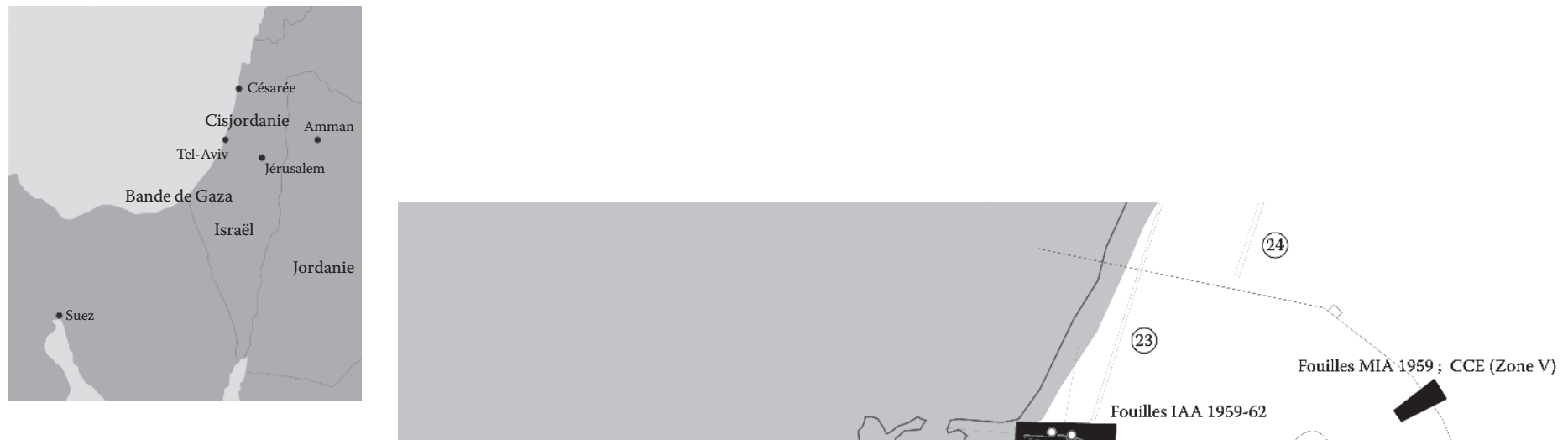

우

[Fig. 1] Carte d'Israël avec localisation de Césarée.

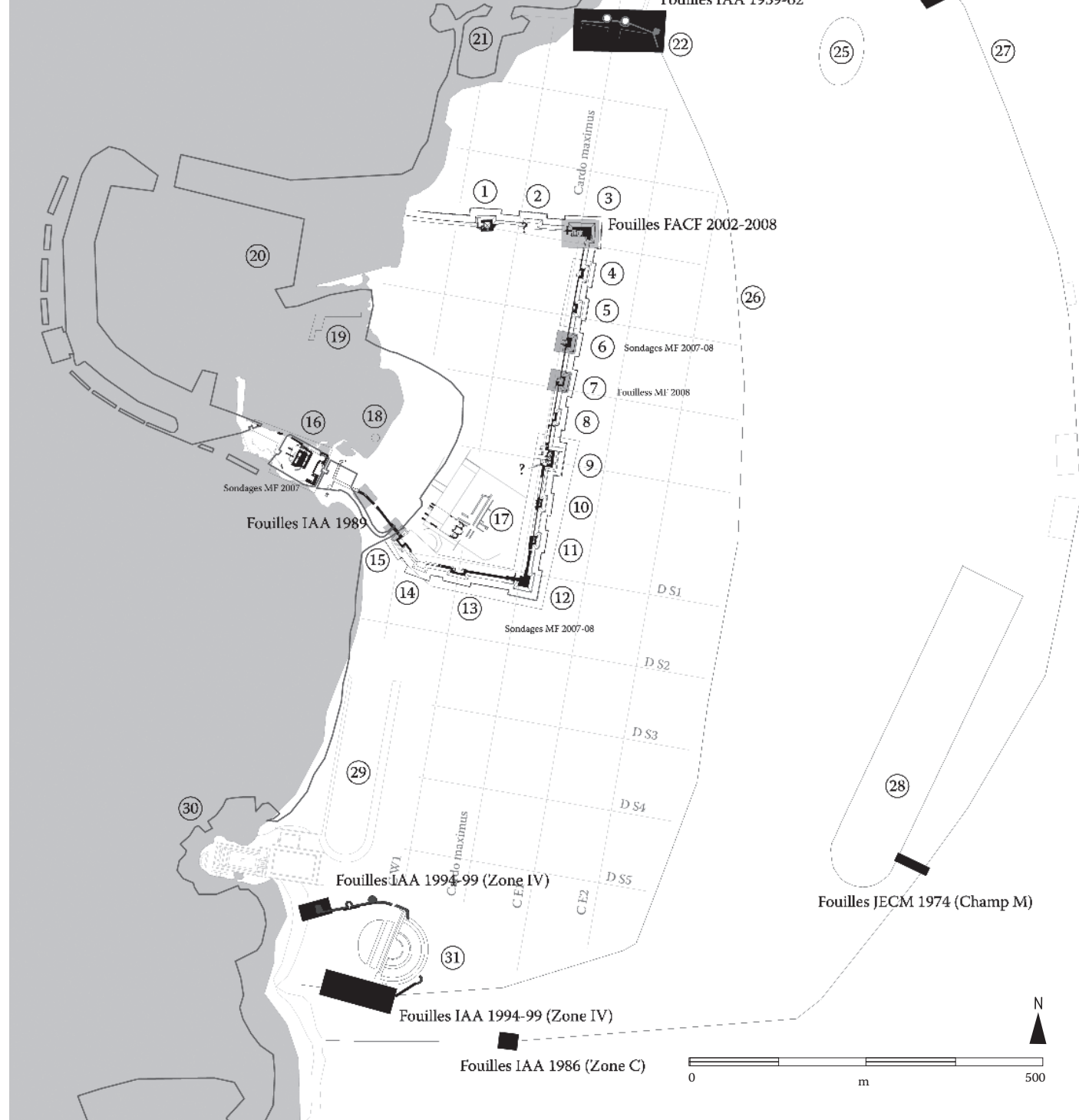

[Fig.2] Plan de l'historique des fouilles

1 - Tour 1 , porte nord

2 - Tour 2

3 - Tour 3

4 - Tour 4

5 - Tour 5

6 - Tour 6

7 - Tour 7

8 - Tour 8

9 - Tour 9, porte est

10 - Tour 10

11 - Tour 11

12 - Tour 12

13 - Tour 13

14 - Tour 14

15 - Tour 15 , porte sud-ouest
16 - Chateau

17 - Cathédrale

18 - Tour immergée

19 - Jetée des colonnes

20 - Avant-Port

21 - Port nord

tour polygonale

23 - Acqueduc

extérieur

24- Acqueduc

intérieur

25 - Amphitheâtre

26 - Enceinte

d'Hérode
22 - Porte nord et
27 - Enceinte

byzantine

28 - Hippodrome

29 - Cirque d'Hérode 30 - Palais d'Hérode

31 - Théâtre et son enceinte 
des campagnes romaines sur le continent européen. Quant au domaine reconstruit au cours du IV siècle, les moyens de subsistance de ses occupants restent inconnus, mais la suite de l'espace bâti au nord n'a jamais été explorée, et doit certainement contenir des réponses à ces questions.

En se consacrant au secteur dédié à l'ornementation végétale, ces campagnes ont apporté des informations qui intéressent la compréhension de la résidence de Jebel Oust. Les quelques résultats présentés ici montrent qu'en s'éloignant des aspects strictement architecturaux et en se penchant sur des secteurs marginaux, la compréhension des vestiges les plus spectaculaires du site s'améliore. L'exploitation de l'ensemble des données permettra de présenter un exemple richement documenté d'habitat rural de l'Afrique romaine.

\footnotetext{
BEN AbEd A., SCHEID J., 1905, « Nouvelles recherches archéologiques à Jebel Oust (Tunisie) », in CRAI, p. 321-349.

Ben BASSEn H., MAurin L. (ÉD.), 1998, Oudhna (Uthina). La Redécouverte d'une ville antique de Tunisie, Bordeaux, Paris, Tunis, p. 212.

Carton Dr., 1907, Bulletin de la Société Archéologique de Sousse, p. 22-23. CARTON DR., 1908, Comptes-rendus de l'Association française pour l'avancement des sciences, Congrès de Clermont-Ferrand.

CARTON Dr., 1914, Revue Tunisienne, p. 248.

FENDRI M., 1963, «Évolution chronologique et stylistique d'un ensemble de mosaïques dans une station thermale à Djebel Oust (Tunisie) », in La Mosä̈que gréco-romaine, colloque du CNRS, Paris, 1965, p. $157-173$.

Maurin L., 2003, Bir Mcherga 028 (S. Ben Baaziz, Carte Nationale des sites archéologiques et des monuments historiques), Tunis, p. 112
}

\section{Programme d'étude quadriennal des fortifications médiévales de Césarée}

\author{
Jocelyn Martineau \\ Inrap \\ Jean Mesqui \\ Société française d'archéologie \\ Nicolas Faucherre \\ Université de Nantes
}

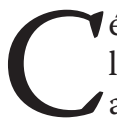

ésarée, Caesarea Maritima, est située sur la côte méditerranéenne d'Israël, à $60 \mathrm{~km}$ au nord de Tel-Aviv et à $40 \mathrm{~km}$ au sud d'Acre [Fig.1]. Devenue, depuis une vingtaine d'années, l'un des sites archéologiques les plus visités d'Israël, elle a été le siège d'une activité archéologique considérable à partir des années 1950, visant à révéler son passé antique et médiéval. Les fouilles menées entre les années 1960 à 2000 se sont concentrées

1 Professeur à l'institut d'archéologie, Université hébraïque de Jésuralem. au-delà du périmètre strictement hérodien; et enfin, à la période franque (entre 1251et 1252), la construction par Louis IX d'une enceinte quadrangulaire rétractée sur le port, seule subsistante de l'œuvre considérable de ce roi pour protéger les ports de la côte. La délimitation de la ville du Haut-Moyen Âge, après la conquête arabe au $\mathrm{VII}^{\mathrm{e}}$ siècle, restait à préciser, même si les fouilles menées à l'ouest du théâtre montraient un secteur urbain vivace à cette période. Le dégagement de la dernière enceinte, celle de saint Louis, dans les années 1960, a montré qu'elle constituait en fait une refortification d'une enceinte plus ancienne de même tracé. Elle fut d'abord attribuée aux Francs et datée de la période suivant leur conquête (1101). Mais plusieurs observations d'Israël Levine, et des découvertes archéologiques ponctuelles faites par Joseph Porath, au sud de l'enceinte franque, ont amené les archéologues israéliens à supposer que l'enceinte primitive existait déjà à l'époque islamique ancienne ; cependant, les indices étaient trop ténus pour la dater précisément comme pour la replacer dans son contexte urbain. La seule intervention archéologique programmée ensuite remonte à 2004 et portait sur un élément de courtine de la tour 3 .

La mission française de Césarée, menée entre 2007 et 2010, avait donc pour objectif général de préciser le plan et la chronologie de cette troisième enceinte essentiellement attribuée aux Francs. Le financement de l'opération a reposé sur la subvention du ministère français des Affaires étrangères et européennes, complétée par des subventions du Centre d'études supérieures de civilisation médiévale de l'Université de Poitiers (CESCM) et du Centre de recherche français de Jérusalem (CRFJ). Une convention de coopération, entre l'Institut national de recherches archéologiques préventives (Inrap) et la direction des Antiquités israéliennes (IAA), a par ailleurs permis de bénéficier de l'assistance de deux collègues de l'Inrap (un archéologue spécialiste du bâti médiéval et un topographe).

\section{Déroulement et résultats des campagnes.} Sur un linéaire d'un kilomètre de longueur, il était nécessaire d'adopter une approche pragmatique de l'enceinte médiévale de Césarée, une fouille systématique n'étant pas envisageable. Elle l'était d'autant moins que l'enceinte était archéologiquement très mal connue. Nous avons opté pour une approche double reposant, d'une part, sur le relevé systématique des structures en place au-dessus du sol (comprenant la topographie, le relevé architectural et l'analyse des maçonneries) et, d'autre part, sur la fouille exhaustive de deux tours de l'enceinte, les tours 6 et 7 . Le choix de ces deux ouvrages, effectué après la campagne 2007, ne tient ni au hasard, ni à l'opportunité; en effet, la tour 6 était la seule de l'enceinte à présenter de façon manifeste une élévation interne antérieure à Louis IX, alors que la tour 7 offrait une structure complexe qui autorisait à envisager le processus constructif. du temple et la frange littorale occidentale

d'un quartier byzantin et arabe ancien [Fig.2].

Bien que l'enceinte médiévale de la ville ait été reconnue depuis le $\mathrm{XIX}^{\mathrm{e}}$ siècle, ces fortifications nont pas été une priorité des recherches depuis leur dégagement sous la direction de Avraham Negev (entre 1959 et 1963). À ce moment, l'histoire des fortifications de Césarée se résumait à trois périodes d'aménagement : à la fin du i ${ }^{\mathrm{er}}$ siècle avant notre ère, la première ligne de défense, élevée sous Hérode, avec la fondation de la première ville; entre le $\mathrm{v}^{\mathrm{e}}$ et le $\mathrm{VI}^{\mathrm{e}}$ siècle, à la période byzantine tardive, la création d'une grande enceinte ovalaire 


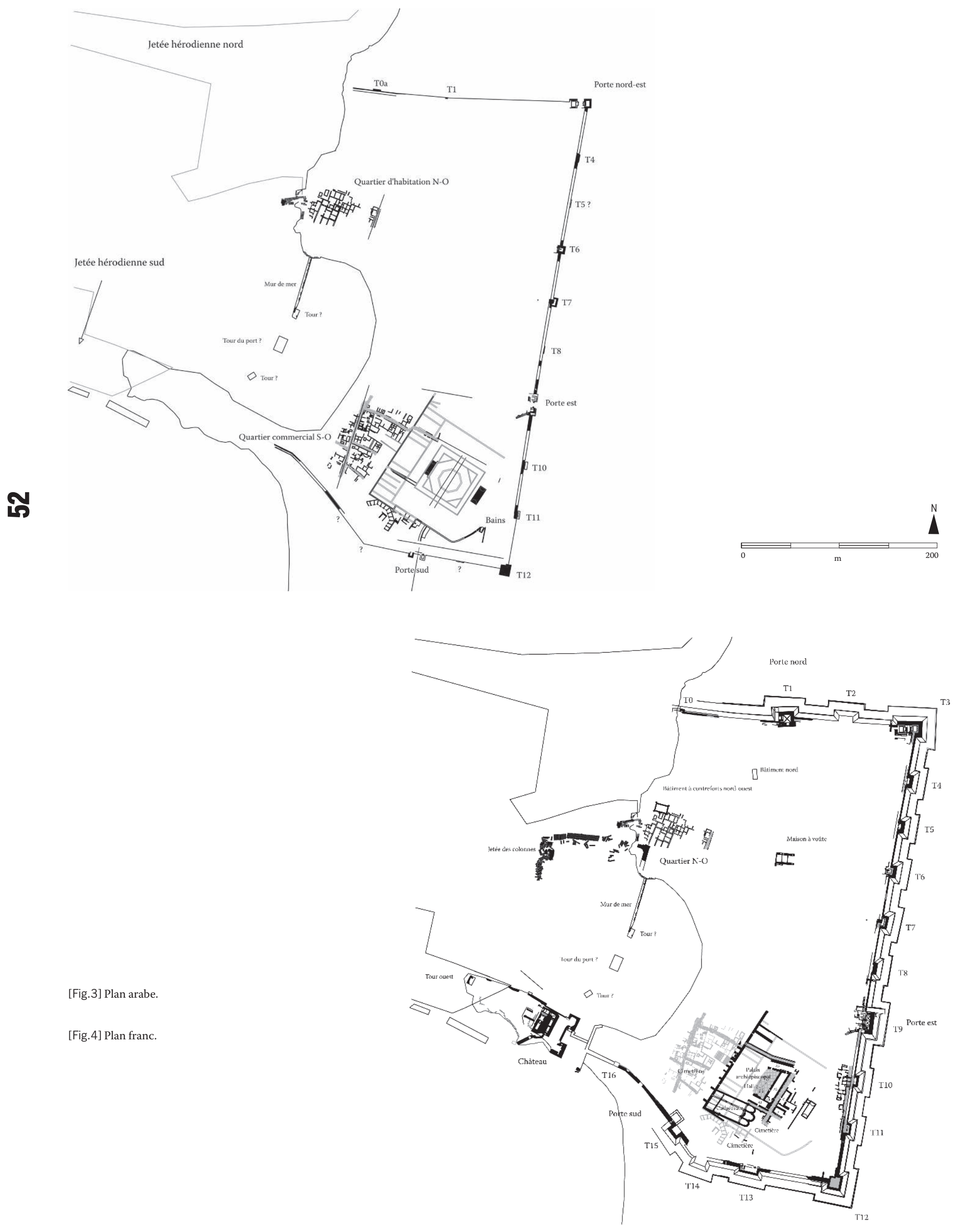


La première campagne d'étude ${ }^{2}$ visait donc à reconnaître le potentiel archéologique de l'enceinte par un relevé topographique et architectural de l'ensemble des tours, des courtines ainsi que des restes du château. Il a été possible ainsi de dresser, pour la première fois, un plan archéologique du secteur du château [Fig.3 et 4] et d'identifier des éléments architecturaux d'importance : une tour primitive à bossages, pourvue d'un couloir d'entrée à trois herses successives, datable, en l'état actuel des recherches, de 1218 ou 1228 ; les ruines de la grande salle voûtée d'ogives construite par saint Louis au-dessus de la tour primitive; les traces du « grand degré » appuyé contre sa face ouest pour desservir cette grande salle. Les relevés ont aussi permis de discerner les vestiges de courtines et de tours de flanquement d'une enceinte primitive englobée dans la fortification de saint Louis. L'hypothèse d'une clôture continue de même tracé que l'enceinte actuelle a été confortée.

La campagne suivante ${ }^{\mathbf{3}}$ a été consacrée à la compréhension des conditions d'implantation de l'enceinte primitive de Césarée sur le cardo de la ville byzantine. Les niveaux d'occupation anciens, sous les fondations de la fortification médiévale, ont été identifiés lors de sondages à l'intérieur et aux abords de deux tours situées au nord-est de l'enceinte. La tour 6 est l'une de celles où l'enchemisement d'une tour primitive par l'enceinte de saint Louis était le plus manifeste. La tour 7 présentait l'avantage d'offrir au moins quatre phases d'occupation successives, datables de la période byzantine à la période franque. Enfin, ces deux tours offraient l'avantage d'être situées dans une zone proche de la fouille réalisée par Joseph Porath sur la tour 3, permettant à terme des mises en relation des résultats acquis par chacun des chantiers de fouilles.

L'objectif de la troisième campagne ${ }^{4}$ fut donc de préciser la chronologie de l'enceinte depuis sa fondation jusqu'à l'époque de saint Louis, en concentrant l'intervention sur les tours 6 et 7 . Il importait de mieux cerner le contexte de fondation et d'appréhender les diverses phases de modification dans la structure tant des fortifications que de leur environnement.

Lors de la dernière campagne ${ }^{\mathbf{5}}$, nous avons achevé la fouille de l'intérieur de la tour 6 ainsi

\footnotetext{
2 Mesqui, Nicolas Faucherre, Bruno Merlin (architecte), Eyal Tamir (topographe israélien) et Hervé Barbé (archéologue à la Direction des Antiquités Israéliennes). 3 Même équipe, rejointe par Jocelyn Martineau et Fabien Sanz-Pascual (Inrap), Kate Rafael (archéologue bénévole israélienne) et Nicolas Morelle (étudiant en archéologie médiévale à l'Université de Rennes).

de Fabien Briand et

de Philippe Boeckler

de Nantes).

5 Avec le concours

de Jean-Philippe Jouan

du patrimoine)

6 Professeur associé

en géographie historique,

Université de Jérusalem.

Université de Haïfa.

et d'études à l'IAA.
}

que les relevés architecturaux des autres tours, entamés en 2007, et réalisé le relevé des profils de moulures et de nervures de l'ensemble du site. Élévations, coupes et plans issus des deux secteurs de fouille ont été systématiquement relevés manuellement par Fabien Sanz-Pascual au 1/20. Les campagnes de fouilles ont ainsi fourni au total 75 minutes de terrain. L'ensemble du mobilier recueilli a été lavé, conditionné et inventorié par Hervé Barbé et l'étude du corpus céramique est en cours. Une table-ronde organisée le 20 mai au Centre de recherche français de Jérusalem (CRFJ), en collaboration avec Ronnie Ellenblum ${ }^{6}$, Joseph Patrich ${ }^{7}$ et le CRFJ, sous la direction de Gideon Avni ${ }^{\mathbf{8}}$, a été l'occasion de faire le point sur la recherche archéologique menée sur le site de Césarée ces vingt dernières années en présentant nos résultats dans un cadre scientifique élargi.

\section{Les principaux constats de l'étude} archéologique du bâti. L'analyse archéologique du château a révélé un édifice complexe, pourvu d'un passage d'entrée remarquable et sans équivalent, présentant une grande salle voûtée sur ogives [Fig.5], accessible par un grand degré dont on ne trouve d'exemple analogue qu'à l'hôpital d'Acre. L'ensemble fortifié, artificiellement séparé de la terre ferme par un fossé, était entouré d'une enceinte flanquée de tours résultant de plusieurs campagnes de construction, et notamment de fortifications construites entre les $\mathrm{XII}^{\mathrm{e}}$ et XIII ${ }^{\mathrm{e}}$ siècles. La mission a également montré que l'imposante enceinte en avait entièrement englobée une plus ancienne, flanquée de tours et de contreforts, existant dès la période islamique ancienne.

\section{La tour-porte est}

La tour-porte est ( $\left.\mathrm{T}_{9}\right)$, ouvrage emblématique de l'enceinte franque, est un exemple de cette reprise, par les Francs, de structures islamiques [Fig.7]. Depuis les dégagements des années 1960-70, elle constitue l'entrée principale de la ville close. Elle a fait l'objet du plus grand nombre de restaurations. Consistant à remonter les voûtes de la salle d'entrée franque, ces travaux sont assez bien documentés par les photographies anciennes, ainsi que par une coupe d'Avraham Negev montrant l'état avant travaux. L'analyse précise des maçonneries montre l'extrême discernement de Negev et le soin avec lequel il a maintenu intacts tous les indices archéologiques repérés. On notera qu'il a également laissé en place les sols au-dessous du niveau XIX ${ }^{\mathrm{e}}$ siècle, à l'intérieur de la tour, ce qui devrait permettre une fouille future de celle-ci. Il s'agit d'une restauration exemplaire; le seul regret que l'on peut avoir porte sur l'absence totale de publication des découvertes qu'il fit pendant les travaux, si l'on excepte un article manuscrit où seules les premières observations de l'archéologue furent manifestement consignées. Il s'agit d'une tour-porte rectangulaire de 22,25 $\mathrm{m}$ de largeur frontale, pour 8,63 $\mathrm{m}$ de profondeur hors-murs au nord, et 10,5 $\mathrm{m}$ au sud; elle est enchapée sur ses faces est et sud par le glacis général, qui monte 

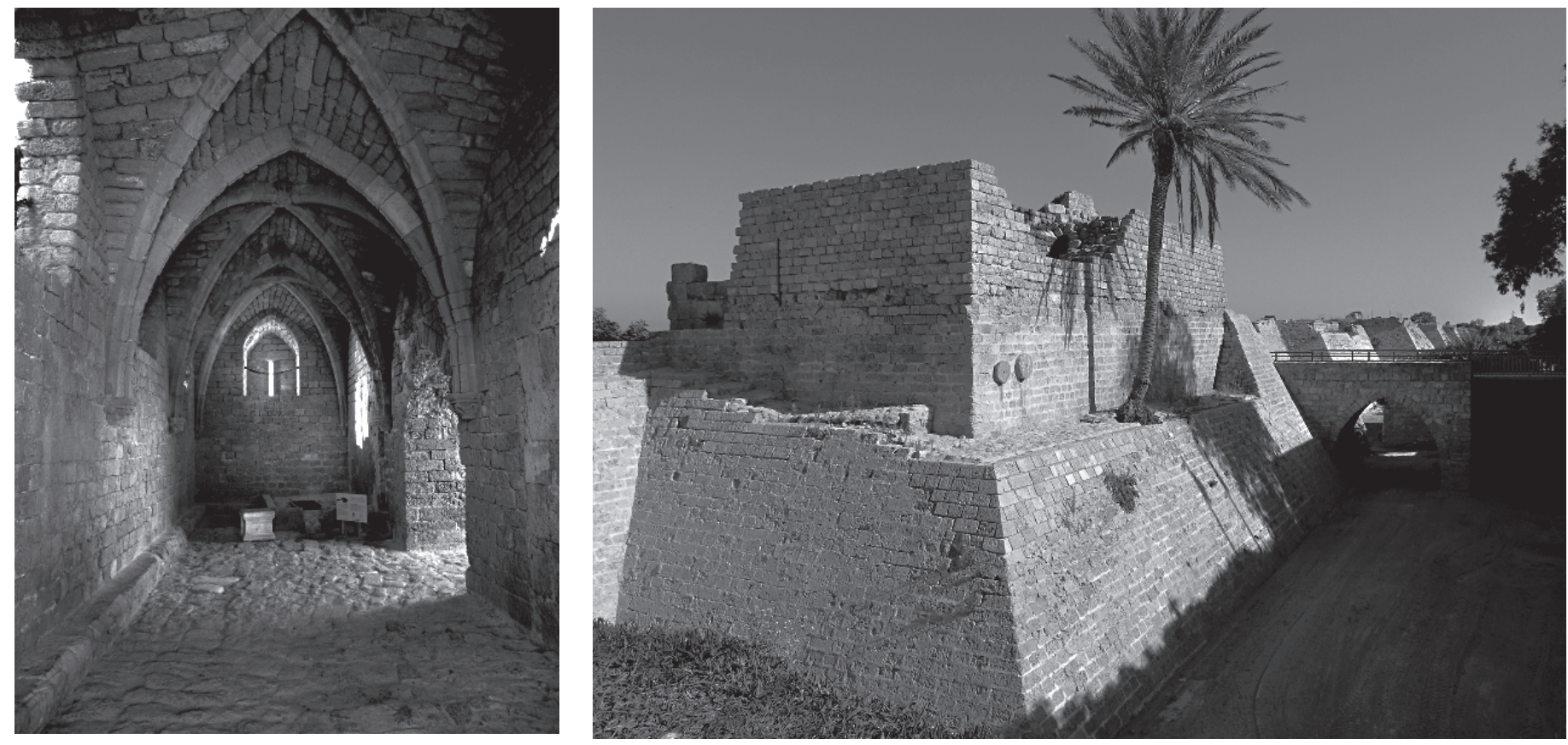

षั
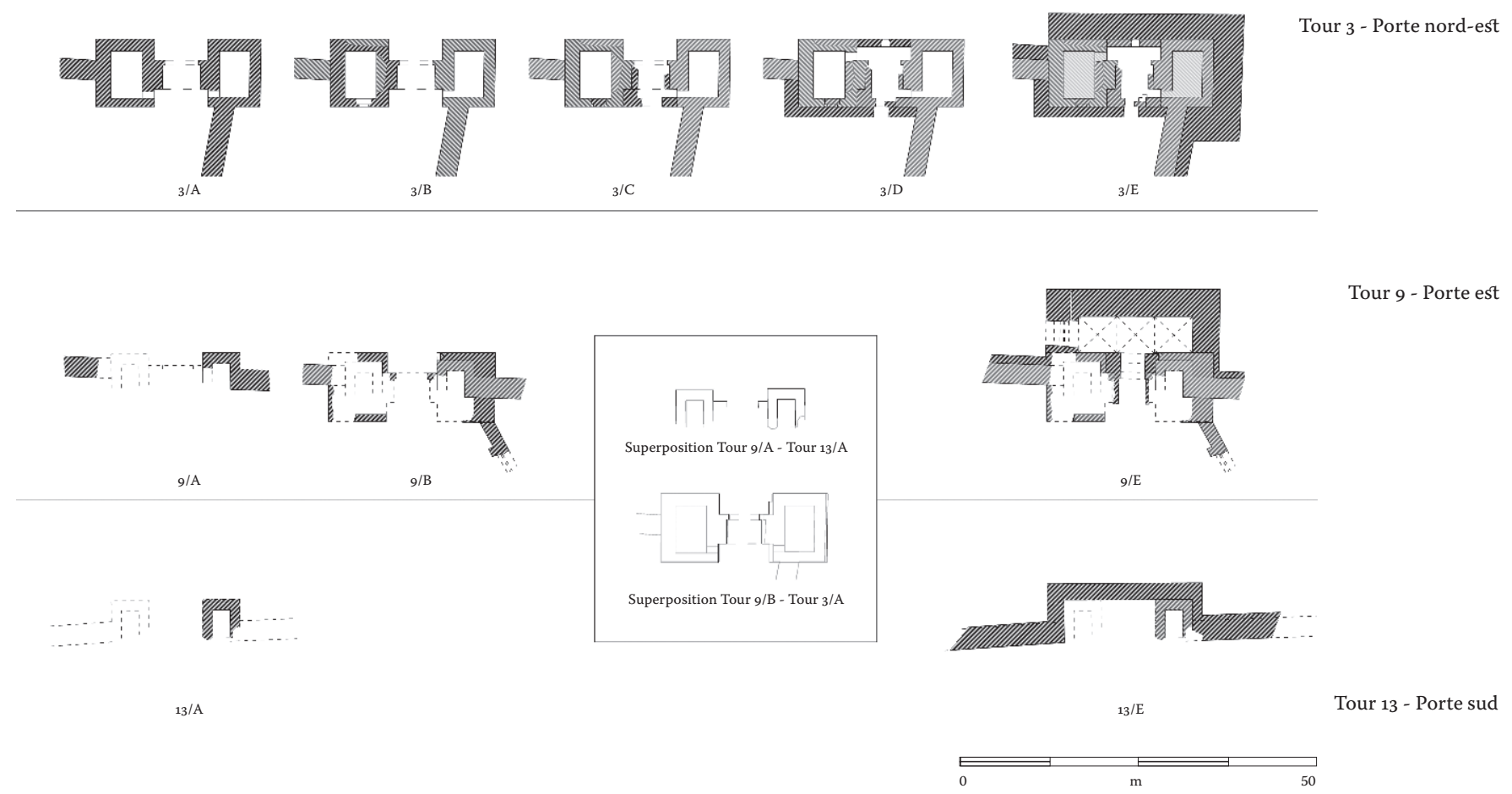

[Fig.5] Tour-porte 10.

[Fig.6] Césarée, tour-porte est.

[Fig.7] Évolution des portes

primitives.

our 3 - Porte nord-est 
ici seulement à 11,6 m ASL. Au nord, elle est bordée par le massif d'accès, prolongé par un pont de maçonnerie à une arche, constituée d'arcs au tracé brisé et supportant un tablier de bois moderne ; le pont ne rejoignait pas la contrescarpe, et il existait donc un dispositif de pont-levis ou de pont mobile pour protéger l'accès. Comme à la tour 1 , le passage d'entrée est doublement coudé: il tourne une première fois sur la gauche, pour passer sous la porte extérieure, au nord. Celle-ci est large de 3,14 m ; l'arc en a été remonté par Negev, mais il en subsistait les piédroits au moment du dégagement. L'entrée dans la salle était contrôlée successivement par un assommoir et par une herse ; au revers, le seuil de marbre est partiellement conservé, ainsi que les crapaudines supérieures des vantaux, qui étaient bloquées par une barre dont subsistent les logements, de part et d'autre du passage. La longue salle était couverte de trois travées de voûtes d'ogives, remontées par Negev en récupérant les claveaux qu'il trouva pendant les dégagements, complétés par des pierres qu'il fit retailler sur le même modèle. Les arcs retombent sur des chapiteaux de très belle facture; le chapiteau d'angle nord-est présente la particularité d'une colonne coudée, suivant une tradition bien ancrée au Proche-Orient. Au fond de la salle, vers le sud, se trouve un bel abreuvoir carré, collé dans l'angle sud-ouest et alimenté par une citerne (peut-être toujours alimentée, si l'on en juge par l'humidité des parements de la paroi ouest). Il s'agit d'une disposition très intéressante dans un ouvrage d'accès et d'une grande fonctionnalité, puisqu'elle permettait aux bêtes et aux hommes de se désaltérer à l'ombre. Le passage se retournait à nouveau, cette fois à droite (vers l'ouest), passant dans un couloir de 3,25 $\mathrm{m}$ de long, dont la voûte a été remontée pour l'essentiel par Negev; cependant, sa coupe prouve que le dessus de la voûte subsistait, traversé par un assommoir qui surveillait le passage. Ce segment était protégé également par des vantaux dont les crapaudines ont été pillées (on voit encore leurs encoches). Puis, au-delà, on empruntait un passage de $6,6 \mathrm{~m}$ de long, peut-être voûté, mais aucun indice ne l'atteste; Negev n'a d'ailleurs pas remonté ce couloir, certainement faute de preuves.

\section{La tour 6}

Dans ce secteur, de nombreux indices laissent penser à une réaffectation rapide, à des fins défensives, d'un quartier de la ville byzantine précédemment abandonnée dans le courant du $\mathrm{VI}^{\mathrm{e}}$-VII ${ }^{\mathrm{e}}$ siècle. Le portique oriental du cardo antique, partiellement ruiné et remblayé sous une couche de terre végétale, est en effet réutilisé comme support d'une enceinte de 2,50 m de large, flanquée d'une tour carrée de 7,57 m de côté horstout $\left(57,30 \mathrm{~m}^{2}\right)$ fondée sur un soubassement plein de $57,30 \mathrm{~m}^{2}$. La colonne découverte sous le mur de gorge occidental, à un niveau plus bas que les deux colonnes précédentes, est particulièrement représentative du système de fondation à colonnes en boutisse que l'on retrouve dans toute l'enceinte, de la période arabe ancienne à la période franque [Fig.9].
Couchée dans un axe nord-sud, il s'agit plus exactement d'un fût monolithe de granit à gros grain bleu-noir d'environ o,70 $\mathrm{m}$ de diamètre. Trois autres colonnes ont été retrouvées couchées dans le sens opposé est-ouest, en travers du cardo, générant ainsi un chaînage original dont le rôle devait être plus antisismique que défensif [Fig.10]. La fouille et l'étude du bâti indiquent que les quatre élévations de la tour ont été édifiées en même temps que les deux courtines adjacentes. Seul le premier niveau subsiste sur toute sa hauteur d'origine, soit 3,50 $\mathrm{m}$, du sol au plancher du premier étage. Le second niveau n'est plus conservé que sur quatre assises, d'une hauteur totale de 1,43 m. Il faut sans doute ajouter l'équivalent jusqu'à hauteur du plancher du deuxième étage disparu. Un troisième niveau est fortement supposé, même s'il n'en subsiste aucune trace. Reprise une première fois par saint Louis, une seconde fois par les Bosniaques avant d'être détruite dans les années 1960, la partie haute de la tour 6 ne conserve plus aujourd'hui que l'arase d'une galerie à quatre archères à niche, d'origine franque. L'examen du parement interne a permis de distinguer deux niveaux de construction, séparés par une césure horizontale à mi-hauteur. Au-dessous de cette césure, les assises ont une hauteur moyenne comprise entre $0,19 \mathrm{~m}$ et $0,28 \mathrm{~m}$, alors qu'au-dessus elles alternent entre assises hautes ( $(0,40$ à $0,46 \mathrm{~m})$ et assises basses (o,20 à o,30 m). Toutes les assises filent d'un mur à l'autre, sans aucune rupture significative. Les angles sont par ailleurs parfaitement chaînés de haut en bas et liés par le même mortier de chaux gris-blanc, ce qui plaide en faveur d'une construction homogène, malgré la différence d'appareillage. Le tout forme un même ensemble architectural homogène, fondé sur le soubassement décrit précédemment. Il est donc probable que la césure ne soit pas significative d'un changement de campagne de construction. La fouille a fait apparaître en outre les restes d'une porte à arc en plein cintre dont il ne subsiste que le piédroit oriental. La stratification progressive des sols d'occupation du rez-de-chaussée a généré une séquence stratigraphique d'une épaisseur totale d'environ o,70 m. Intégralement fouillés, ces sols de terre battue ont livré un mobilier abondant et varié. Les premiers résultats, corroborés par l'analyse au carbone 14 de trois échantillons prélevés dans les mortiers de chaux (charbon de bois et noyau d'olive), ont révélé une datation entre la fin du viI ${ }^{\mathrm{e}}$ siècle et le début du $\mathrm{x}^{\mathrm{e}}$ siècle, c'est-à-dire à l'époque omeyyade ou à l'époque abbasside.

En développant une méthode propre, avec des moyens très modestes adaptés au contexte et aux possibilités financières du MAEE, avec le soutien logistique des autorités locales, la mission a réussi à cerner la chronologie relative des fortifications médiévales de Césarée et à mettre au jour une fortification urbaine islamique ancienne quasi-inédite au Proche-Orient. Le facteur déterminant a sans doute été également la rencontre et la confrontation de connaissances 


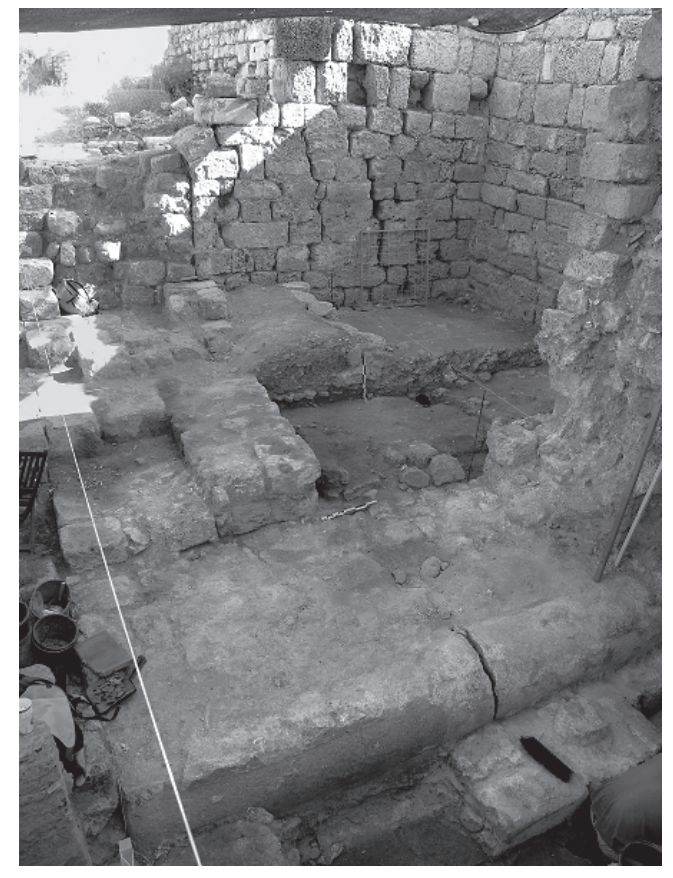

ณீ

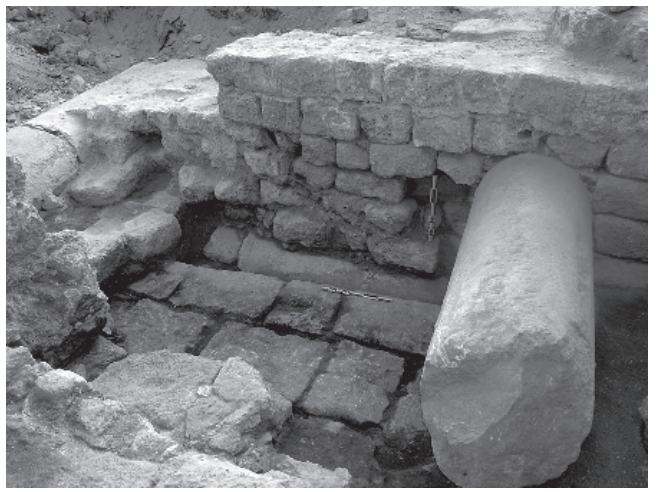

[Fig.8] Tour 6 en 2009.

[Fig.9] Colonne en boutisse.

[Fig.10] Plan de la tour 6 .
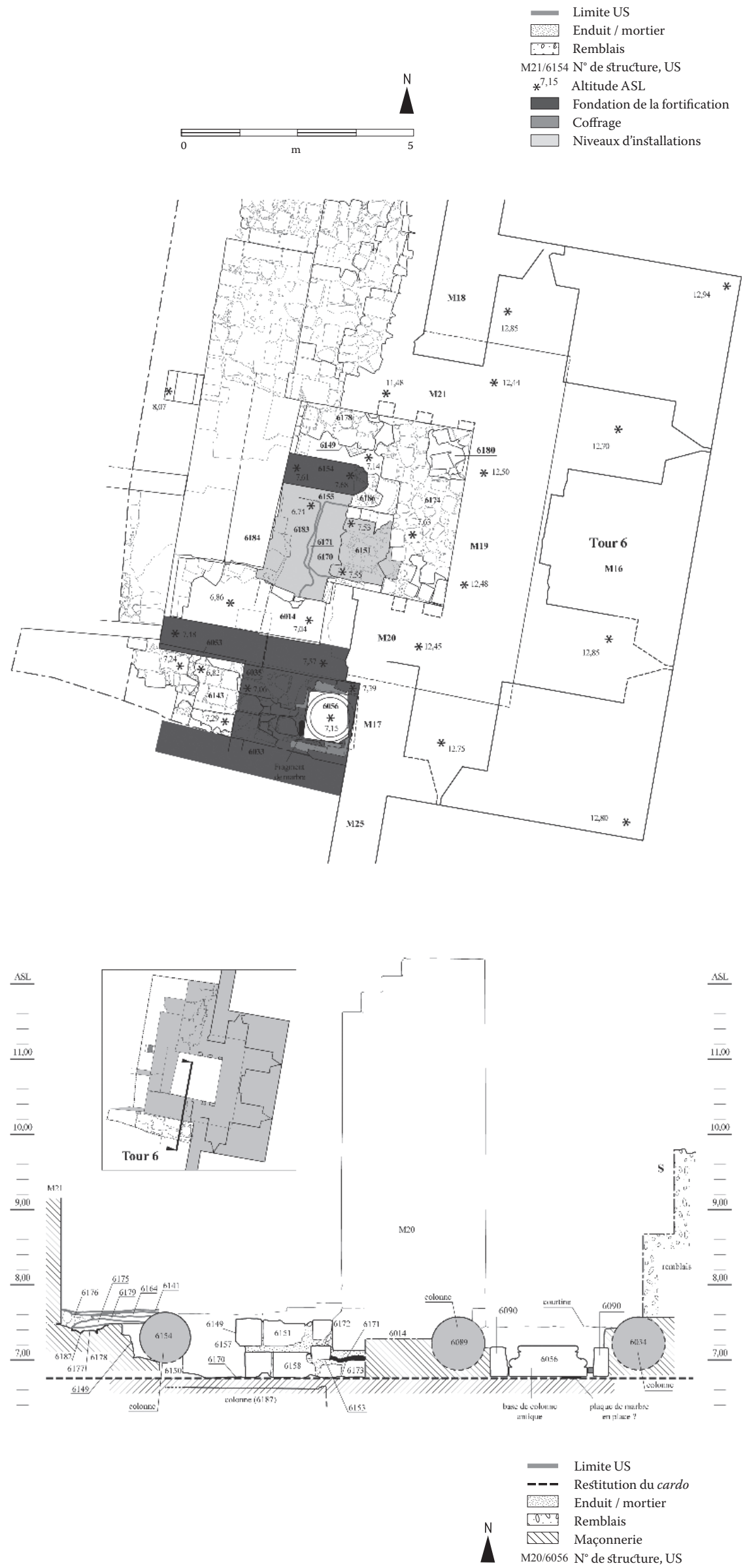
et de pratiques d'archéologues de formation et de pays différents. Il a été particulièrement intéressant pour les chercheurs, tant français qu'israéliens, de faire la part, sur l'un des plus grands sites archéologiques du Proche-Orient, entre les techniques architecturales des Croisés et les réalités du bâti préexistant qui ont été combinées. Cette confrontation a eu ses moments de passion ou de doute, mais elle a permis de se comprendre, d'apprendre à parler et à voir avec les yeux de l'autre sans immédiatement prendre une position dogmatique.

\section{Projet de collaboration franco- canadienne et franco-américaine en archéologie préventive : apport à la connaissance des sites nord- américains d'époque moderne}

\author{
Fabienne Ravoire
}

Inrap

a création d'une session sur la culture matérielle avec Steven Pendery, dans le cadre du IV Congrès international d'archéologie médiévale et moderne, "Medieval Europe in Paris », en septembre 2007, a constitué, à n'en pas douter, la première manifestation d'une collaboration internationale ${ }^{2}$. Un an plus tard, celle-ci se poursuivit par la présentation d'une synthèse sur les céramiques françaises des XVI ${ }^{\mathrm{e}}$ et XVII ${ }^{\mathrm{e}}$ siècles (cf. p. 60) pour le colloque « Histoire et Archéologie des XVI ${ }^{\mathrm{e}}$ et $\mathrm{XVII}^{\mathrm{e}}$ siècles », dans le cadre du CXXXIII ${ }^{\mathrm{e}}$ Congrès international du Comité des travaux historiques et scientifiques (CTHS), dont le thème était «Migrations, transferts et échanges de part et d'autre de l'Atlantique» (Québec, 6-8 juin 2008). Cet article, intitulé « Les céramiques en usage à Paris et dans les grandes villes côtières françaises au XVI ${ }^{\mathrm{e}}$ siècle et au début du XVII ${ }^{\mathrm{e}}$ siècle : contribution à l'identification des productions exportées sur les sites coloniaux nord-américains », proposait un panorama de la céramique en usage en France à cette époque et ainsi de faire le lien avec les mobiliers mis au jour sur les nombreux sites canadiens et nord-américains. Il s'agissait d'examiner le problème de la diffusion et de l'utilisation des céramiques à ces époques,

1 Senior Archeologist, Northeast Region, National Park Service. 2 Section 3 : Culture matérielle : de l'objet à l'individu. l'analyse de leur distribution en France (production, datation et utilisation). Les fouilles de sites relatifs aux premiers temps de la colonisation par les Européens, et notamment les Français, en Amérique du Nord, sont nombreuses depuis ces trente dernières années. Elles concernent d'un côté des établissements fortifiés établis le long de la côte, dont les plus anciens, celui de CartierRoberval à Québec et celui de Charlesfort en Caroline du Sud remontent aux décennies 1540-1560 et les autres au tout début du XVII ${ }^{\mathrm{e}}$ siècle, comme le fort de l'île-Sainte-Croix, Maine (Pendery, 2007 a), l'Habitation de Port-Royal, qui devint la capitale de l'Acadie (Nouvelle-Écosse), l'Habitation de Champlain (Niellont, Moussette, 1985) et la ferme du cap Tourmente à Québec (Guimont, 1996 ; Moussette, Moss, 2008), le fort de Pointe à Callière, à Montréal, mais aussi Jamestown, colonie anglaise (Virginie). Ces fouilles portent, d'un autre côté, sur des sites de pêcheries morutières et de chasse à la baleine, situés en bordure des côtes comme celui de Petit Mécatina (Herzog, à paraître) ou du Petit Nord (Pope, 2007) à Terre-Neuve, ainsi que des épaves retrouvées dans l'estuaire du Saint-Laurent comme celle de Red Bay (Grenier et al., 2007). La plupart de ces sites ont livré quantité d'objets relatifs à la culture matérielle européenne (perles, verreries, pipes, bibelots, etc.) et notamment des céramiques françaises. Les mécanismes d'acquisition et de diffusion de ces dernières reflètent des réalités différentes : vaisselle de bord des marins embarqués pour les pêches dans les eaux de Terre-Neuve, vaisselles personnelles des migrants, enfin, à partir du milieu du XVII ${ }^{\mathrm{e}}$ siècle, quand les établissements furent plus enracinés dans les nouvelles zones conquises, cargaisons de céramiques destinées aux colons.

Sur les sites fortifiés comme dans les épaves, on retrouve des céramiques embarquées pour leur contenu : pots à beurre en grès bas-normand; amphores à huile espagnoles ; céramiques culinaires (cuisson et fonte des graisses animales) d'origine bretonne, béarnaise, du Sud-Ouest et peut-être aussi provençale ; vaisselle de table, notamment des écuelles en faïence lustrée espagnole. Sur les sites de pêcheries, la céramique française se compose des mêmes pots à beurre en grès brun bas-normand, parfois d'amphores espagnoles, mais aussi de récipients en grès gris clair originaires du Beauvaisis et destinés au service des boissons (coupelles, gourdes, pichets), à la conservation des salaisons (saloirs) et des médications (albarelles, flacons). La vaisselle culinaire est également attestée sur la plupart de ces sites, mais son origine reste encore à préciser (Normandie, Saintonge). Les études réalisées sur le site de Cartier-Roberval semblent témoigner d'origines normande et bretonne qui sont en cours de validation par des analyses de provenance (cf. p. 6o). Outre ces récipients d'usage domestique, la plupart des sites, qu'ils soient liés aux activités de pêche où aux zones d'implantation, ont livré des céramiques ornementales : écuelles espagnoles en faïence lustrée, assiettes à glaçure polychrome de dans une perspective économique et sociale. Enfin, en 2008, l'expertise du mobilier céram du site de Cartier Roberval à Cap Rouge, près de français du Canada (1541), fut la première collaboration entre l'Inrap et la Commission de la capitale nationale de Québec (cf. p.6o).

Production et datation de céramiques françaises trouvées sur des sites nordaméricains. Ces travaux reposent, d'une part, sur l'identification des mobiliers français dans les sites canadiens et nord-américains et, d'autre part, sur 